\title{
ON THE ORTHOGONALITY OF MEASURES INDUCED BY L-PROCESSES(1)
}

BY

MAREK FISZ

1. Introduction and summary. Let $\{X(t), 0 \leqq t \leqq 1\}$ be a real, centered stochastic process with independent increments with no fixed points of discontinuity and with $X(0)=0$. The random variable $X(t)$ has then, for any $0 \leqq t \leqq 1$, an infinitely divisible distribution function $F(t, x)$ with characteristic function $\phi(t, v)$ satisfying Lévy's [9] formula

$$
\log \phi(t, v)=i \gamma(t) v-\frac{1}{2} \sigma^{2}(t) v^{2}+\left[\int_{-\infty}^{0-}+\int_{0+}^{\infty} A(u, v) d_{u} H(t, u)\right],
$$

where $\gamma(t)$ and $\sigma(t) \geqq 0$ are continuous functions and $\sigma(t)$ is nondecreasing, $A(u, v)=e^{i u v}-1-i v u /\left(1+u^{2}\right), H(t, u)$ is, for any $t \in[0,1]$, defined and nondecreasing for $u<0$ and $u>0, H(t,-\infty)=H(t,+\infty)=0$ and, for any finite $\varepsilon>0$,

$$
\left[\int_{-\varepsilon}^{0-}+\int_{0+}^{\varepsilon} u^{2} d_{u} H(t, u)\right]<\infty
$$

For any $t \in[0,1]$ and $u<0(u>0)$, the function $H(t, u)(-H(t, u))$ is equal to (see Doob [3, VIII, §7]) the expected number of jumps of the process $X(t)$ before time $t$ of size less than $u$ (larger than $u$ ).

We remind the reader that an infinitely divisible distribution function $F(x)$ is said to belong to the class $L(F \in L)$ if it is a limit, in the sense of weak convergence, of a sequence of distribution functions $F_{n}(x)$ of the form

$$
F_{n}(x)=P\left(\frac{X_{1}+\ldots+X_{n}}{B_{n}}-A_{n}<x\right),
$$

where $\left\{X_{j}\right\}(j=1,2,3, \ldots)$ is a sequence of independent random variables, $B_{n}>0$ and $A_{n}$ are some sequences of constants, and $X_{j} / B_{n}$ is asymptotically constant.

If $F \in L$, the function $H(t, u)$ assigned to $F$ by formula (1), has for every $t \in[0,1]$, at any point $u<0$ and $\mathrm{u}>0$ right and left derivatives in $u$ and $u H^{\prime}(t, u)$ is nonincreasing for $u<0$ and $u>0$, where $H^{\prime}(t, u)$ denotes either the right or the left

Presented to the Society, April 13, 1962; received by the editors December 4, 1961.

(1) Research supported in part by National Science Foundation Grant NSF-G14146 at Columbia University. 
derivative in $u$. The function $H(t, u)$ satisfies for arbitrary $u_{1}<u_{2}<0$ and for arbitrary $0<u_{1}<u_{2}$ the inequality

$$
H\left(t, u_{2}\right)-H\left(t, u_{1}\right) \geqq H\left(t, \frac{u_{2}}{\alpha}\right)-H\left(t, \frac{u_{1}}{\alpha}\right)
$$

for any $0<\alpha<1$. (See Gnedenko and Kolmogorov [5, §30].)

We introduce the following

Definition. The stochastic process $\{X(t), 0 \leqq t \leqq 1\}$ with independent increments will be called a $L$-process if, for any $0 \leqq t \leqq 1$, the distribution function $F(t, x)$ of $X(t)$ belongs to $L$.

By Kolmogorov's [7] theorem, any real stochastic process $\{X(t), 0 \leqq t \leqq 1\}$ induces in the space $\mathfrak{U}$ of real functions a probability measure $P_{X}$, defined on the minimal Borel field $\mathscr{F}$ of subsets of $\mathfrak{A}$, generated by the cylindric sets, i.e., by the sets of all real functions $f(t)$ such that, for $n=1,2,3, \cdots$, and any $t_{1}, \cdots, t_{n}$ from the interval $[0,1]$, the vector $\left\{f\left(t_{1}\right), \ldots, f\left(t_{n}\right)\right\}$ takes on values from Borel sets in the $n$-dimensional Euclidean space.

Let now $P_{1}$ and $P_{2}$ be two measures defined on a Borel field $\mathscr{F}$ from some space $\mathfrak{A}$. The measure $P_{2}$ is said to be absolutely continuous with regard to $P_{1}$ $\left(P_{2} \ll P_{1}\right)$ if, for any set $A \in \mathscr{F}$, the equation $P_{1}(A)=0$ implies $P_{2}(A)=0$. If both $P_{1} \ll P_{2}$ and $P_{2} \ll P_{1}$, the measures $P_{1}$ and $P_{2}$ are called equivalent $\left(P_{1} \sim P_{2}\right)$. The measures $P_{1}$ and $P_{2}$ are said to be orthogonal or mutually singular $\left(P_{1} \perp P_{2}\right)$ if for some $A \in \mathscr{F}$ both of the equations

$$
P_{1}(A)=0, \quad P_{2}(\mathfrak{A}-A)=0
$$

hold.

The question of equivalence and orthogonality of measures in function spaces has attracted much attention. The pioneering work is due to Kryloff and Bogoliuboff [8]. An important result is due to Kakutani [6] who has shown that if $P_{i}$ $(i=1,2)$ is a probability measure induced by a sequence of independent random variables $X_{i j}(j=1,2, \cdots)$ and for every $j$ the probability measures of $X_{1 j}$ and $X_{2 j}$ are equivalent, then either $P_{1} \sim P_{2}$ or $P_{1} \perp P_{2}$. The problem of equivalence and orthogonality of measures induced by Gaussian processes has been discussed by Cameron and Martin [2], Prohorov [10], Baxter [1] and Feldman [4]. Necessary and sufficient conditions for the relation $P_{2} \ll P_{1}$ when $P_{1}$ and $P_{2}$ are probability measures induced by processes with independent increments whose parameter range is finite have been given by Skorohod [11]. It is the purpose of this note to give conditions for $P_{X_{1}} \perp P_{X_{2}}$ when $P_{X_{1}}$ and $P_{X_{2}}$ are induced by centered $L$-processes with finite parameter range. It is shown, in particular, that if the $L$-processes are stable processes with unequal $H(t, u)$ functions, then $P_{X_{1}} \perp P_{X_{2}}$.

2. Theorems and proofs. Let $\left\{X_{i}(t), 0 \leqq t \leqq 1\right\}(i=1,2)$ be $L$-processes with $H_{i}(t, u)$ in formula (1). If, for some $t$ and $-\infty<u<0$ or $0<u<\infty$, both 
$H_{1}(t, u)$ and $H_{2}(t, u)$ are identically 0 , we shall agree to say that, on the considered half-line in the plane $(t, u), H_{2}^{\prime}(t, u) / H_{1}^{\prime}(t, u)=1$. We shall prove the following theorems.

THEOREM 1. Let $P_{X_{1}}$ and $P_{X_{2}}$ be probability measures induced in the space of real functions by the centered L-processes $\left\{X_{1}(t), 0 \leqq t \leqq 1\right\}$ and $\left\{X_{2}(t)\right.$, $0 \leqq t \leqq 1\}$ with no fixed points of discontinuity, with $X_{1}(0)=X_{2}(0)=0$ and with $\gamma_{i}(t), \sigma_{i}(t)$ and $H_{i}(t, u)(i=1,2)$ in formula $(1)$. Let $H_{i}^{\prime}(t, u)(i=1,2$,$) denote$ the left-hand and right-hand derivatives in $u$ of $H(t, u)$ for $u<0$ and $u>0$, respectively. If, for some $t_{0} \in(0,1], H_{1}\left(t_{0}, u\right)$ and $H_{2}\left(t_{0}, u\right)$ are not identically 0 , the limits in (3) and (4), finite or infinite, exist, and at least one of the relations

$$
\begin{aligned}
& \rho_{-}\left(t_{0}\right)=\lim _{u \uparrow 0-} \frac{H_{2}^{\prime}\left(t_{0}, u\right)}{H_{1}^{\prime}\left(t_{0}, u\right)}=1, \\
& \rho_{+}\left(t_{0}\right)=\lim _{u \downarrow 0+} \frac{H_{2}^{\prime}\left(t_{0}, u\right)}{H_{1}^{\prime}\left(t_{0}, u\right)}=1
\end{aligned}
$$

does not hold, then $P_{X_{1}} \perp P_{X_{2}}$.

THEOREM 2. Let $P_{X_{1}}$ and $P_{X_{2}}$ have the same meaning as heretofore. If $X_{1}(t)$ and $X_{2}(t)$ are centered, stable processes and, for some $t_{0} \in(0,1], H_{1}\left(t_{0}, u\right) \not \equiv$ $H_{2}\left(t_{0}, u\right)$, then $P_{X_{1}} \perp P_{X_{2}}$.

The proof of Theorems 1 and 2 will be preceded by the proof of three lemmas concerning the $H$ function of $F \in L$. For the sake of brevity, we shall write in the formulation of the lemmas and their proofs $H(u)$, without referring to the argument $t$.

Lemma 1. Let the distribution function $F \in L$ and let $H(u)$ correspond to $F$ by formula (1). Then for $u<0(u>0)$ the relation

$$
\lim _{u \uparrow 0-} H(u)=\infty\left(\lim _{u \downarrow 0+} H(u)=-\infty\right)
$$

holds, unless $H(u) \equiv 0$ for $u<0(u>0)$.

Proof of Lemma 1. Suppose that $H(u) \not \equiv 0$ for $u<0$ and that relation (5) does not hold. Since $H(u)$ is nondecreasing,

$$
\lim _{u \uparrow 0-} H(u)=a<\infty,
$$

and, by the continuity of $H(u)$, it would be possible to find for arbitrary $\varepsilon>0$ and $\eta>0$ two numbers $u_{1}<u_{2}<0$ such that $\left|u_{1}\right|<\eta$ and $H\left(u_{2}\right)-H\left(u_{1}\right)<\varepsilon$. Since $\eta$ is arbitrary, it would then follow from formula (2) that the increment of $H$ on an arbitrary large interval $\left[u_{1} / \alpha, u_{2} / \alpha\right]$ is less than $\varepsilon$. Taking into account that 
$\varepsilon>0$ may be arbitrarily small, we would get $H(u) \equiv 0$ for $u<0$, contrary to the assumption; relation (5), therefore, holds.

The case of $u>0$ may be proved in the same way.

LEMMA 2. Let $F \in L$ anu let $H(u)$ be the function assigned to $F$ by formula (1). If, at some point $u_{0}<0\left(u_{0}>0\right), H\left(u_{0}\right)>0\left(H\left(u_{0}\right)<0\right)$, the function $H(u)$ is for all $u_{0} \leqq u<0\left(0<u \leqq u_{0}\right)$ strictly increasing.

Proof of Lemma 2. Let $H\left(u_{0}\right)>0$ at $u_{0}<0$ and suppose that at two points $u^{\prime}$ and $u^{\prime \prime}\left(u_{0} \leqq u^{\prime}<u^{\prime \prime}<0\right)$ the equality $H\left(u^{\prime}\right)=H\left(u^{\prime \prime}\right)$ holds. Since $H(u)$ is nondecreasing this would imply $H(u)=$ const. for $u^{\prime} \leqq u \leqq u^{\prime \prime}$.

Now relation (2) implies that for any $u_{1}, u_{2}$ such that $u_{1}<u_{2}<u^{\prime \prime}$ and $u_{2}-u_{1}$ $=u^{\prime \prime}-u^{\prime}$ the inequality

$$
H\left(u^{\prime \prime}\right)-H\left(u^{\prime}\right) \geqq H\left(u_{2}\right)-H\left(u_{1}\right)
$$

holds. Indeed, suppose for the moment that $u^{\prime}<u_{2}$ and take $\alpha=u^{\prime \prime} / u_{2}$. We have then by (2)

$$
H\left(u^{\prime \prime}\right)-H\left(u^{\prime}\right) \geqq H\left(u_{2}\right)-H\left(\frac{u^{\prime} u_{2}}{u^{\prime \prime}}\right) \geqq H\left(u_{2}\right)-H\left(u_{1}\right) .
$$

If we drop the assumption $u^{\prime}<u_{2}$, we arrive at (7) by repeating the argument a finite number of times.

Take now points $u_{1}<u_{0}<u_{2}<\cdots<u_{k}<u^{\prime}<u_{k+1}<u^{\prime \prime}<0$ such that $u_{j}-u_{j-1}=u^{\prime \prime}-u^{\prime}(j=2, \cdots, k+1)$. Since $H(u)$ is constant for $u^{\prime} \leqq u \leqq u^{\prime \prime}$, the same will, by relation (7), be true for the interval $\left[u_{1}, u^{\prime \prime}\right]$. Since we can extend this procedure to any interval $\left[a, u^{\prime \prime}\right]$ with $a<u_{0}$, the increase of $H(u)$ on an arbitrary large interval $\left(a, u^{\prime \prime}\right)$ would be equal 0 , contrary to the assumption that $H\left(u_{0}\right)-H(-\infty)>0$.

For $u>0$ the proof runs along the same lines.

LEMMA 3. Let $H(u)$ be the function assigned to $F \in L$ by formula (1). Then $H(u)$ is an absolutely continuous function on $(-\infty, 0-)$ and on $(0+, \infty)$.

Proof. For the proof it is sufficient to show that $H(u)$ is absolutely continuous on any interval $[a, b]$ with $a<b<0$ or $0<a<b$. Let $a<b<0$ and $H(b) \neq 0$. For an arbitrary $\varepsilon>0$ take $c<b$ such that $H(b)-H(c)<\varepsilon$. Put $\delta=b-c$ and consider the disjoint intervals $\left(a_{i}, b_{i}\right)(i=1,2, \cdots, n)$ with $a \leqq a_{1}<b_{1}<a_{2}<b_{2}$ $<\cdots<a_{n}<b_{n} \leqq b$ such that $\sum_{i=1}^{n}\left(b_{i}-a_{i}\right)<\delta$ and otherwise arbitrary. By (7) we get

$$
\sum_{i=1}^{n}\left[H\left(b_{i}\right)-H\left(a_{i}\right)\right]<\varepsilon .
$$

Since $n$ is arbitrary, the absolute continuity of $H(u)$ on $[a, b]$ has been proved. For intervals on the half-line $u>0$, the proof is analogous. 
Proof of Theorem 1. We remark first of all that, without restricting the generality of our considerations, we may assume that $X_{1}(t)$ and $X_{2}(t)$ are separable. Indeed, if they were not separable, we would consider the separable processes $X_{1}^{*}(t)$ and $X_{2}^{*}(t)$ that are stochastically equivalent to $X_{1}(t)$ and $X_{2}(t)$, repectively. (See Doob [3, p. 57].) Since, for $i=1,2$, the finite dimensional distributions of $X_{i}(t)$ and $X_{i}^{*}(t)$ are identical, the probability measure $P_{X_{i}^{*}}$ induced in $\mathfrak{A}$ by $X_{i}^{*}(t)$ is equal to $P_{X_{i}}$ for any set $A$ from $\mathscr{F}$. Therefore, to show that $P_{X_{1}} \perp P_{X_{2}}$ it would be enough to show that for some set $A$ from $\mathscr{F}$ both of the equations $P_{X_{1}^{*}}(A)=0, P_{X_{2}^{*}}(A)=1$ hold. We therefore assume, in the proofs of Theorems 1 and 2, that $X_{1}(t)$ and $X_{2}(t)$ are separable and, in proving orthogonality, we shall use sets $A \in \mathscr{F}$ only.

We remark now that the sample functions of a centered, separable $L$-process $\{X(t), 0 \leqq t \leqq 1\}$ with no fixed points of discontinuity and with $H\left(t_{0}, u\right) \not \equiv 0$ for some $t_{0} \in(0,1]$ are discontinuous, with probability 1 . Indeed, let $N_{n}$ denote the number of jumps before $t_{0}$ either of size $\in\left(-2^{-n},-2^{-n-1}\right]$ or of size $\in\left(2^{-n-1}, 2^{-n}\right]$. Then the $N_{n}$ form a sequence of independent Poisson variables with

$$
\lambda_{n}=E\left(N_{n}\right)=H\left(-2^{-n-1}\right)-H\left(-2^{-n}\right)+H\left(2^{-n}\right)-H\left(2^{-n-1}\right) .
$$

By Lemma 1, we have $\sum_{n=1}^{\infty} \lambda_{n}=\infty$. This implies (see [3, p. 115, Theorem 2.7 (ii)]) that $\sum_{n=1}^{\infty} N_{n}=\infty$, with probability 1 .

Let now $H_{1}\left(t_{0}, u\right)$ and $H_{2}\left(t_{0}, u\right)$ not be identically 0 . Denote by $N_{i}\left(t_{0}, 0-\right)$ and $N_{i}\left(t_{0}, 0+\right)(i=1,2)$ the number of jumps before $t_{0}$ of the process $X_{i}(t)$, of negative and positive size, respectively. If $H_{1}\left(t_{0}, u\right) \equiv 0$ for $u<0$ and $H_{2}\left(t_{0}, u\right)$ $\equiv 0$ for $u>0$, we have $E N_{1}\left(t_{0}, 0-\right)=E N_{2}\left(t_{0}, 0+\right)=0$. Since the sample functions of $X_{1}(t)$ and $X_{2}(t)$ are discontinuous, the $X_{1}(t)$ and $X_{2}(t)$ processes are entirely concentrated on functions with negative and positive jumps, respectively. Hence $P_{X_{1}} \perp P_{X_{2}}$. Denote for $i=1,2$

$$
a_{i}=a_{i}\left(t_{0}\right)=\inf \left\{u: u<0, H_{i}\left(t_{0}, u\right)>0\right\}
$$

and suppose now that both $a_{1}<0$ and $a_{2}<0$. Make the unrestrictive assumption that $a_{2} \leqq a_{1}$. We shall show that for any $u_{1}, u_{2}$ with $a_{1} \leqq u_{1}<u_{2} \leqq 0-$

$$
\int_{u_{1}}^{u_{2}} d_{u} H_{2}\left(t_{0}, u\right)=\int_{u_{1}}^{u_{2}} \frac{H_{2}^{\prime}\left(t_{0}, u\right)}{H_{1}^{\prime}\left(t_{0}, u\right)} d_{u} H_{1}(t, 0 u) .
$$

To see this, let us notice first that, by Lemma $2, H_{1}^{\prime}\left(t_{0}, u\right)>0$ for $a_{1}<u<0$. Next, the derivatives of $H_{1}\left(t_{0}, u\right)$ in $u$ exist everywhere, except possibly at points $u$ belonging to a set of Lebesgue measure 0 . By Lemma 3, this exceptional set has $H_{1}$-measure equal to $0_{j}$ thus relation (8) holds.

Let us now assume that relation (3) does not hold; hence $\rho_{-}=\rho_{-}\left(t_{0}\right) \neq 1$. Suppose $\rho_{-}<\infty$. For $\varepsilon>0$ arbitrary, we could then find a $c<0$ such that for all $u$ from $[c, 0)$ 


$$
\left|\frac{H_{2}^{\prime}\left(t_{0}, u\right)}{H_{1}^{\prime}\left(t_{0}, u\right)}-\rho_{-}\right| \leqq \varepsilon
$$

Take $\varepsilon=(1 / 2)\left|1-\rho_{-}\right|$and a number $c$ such that (9) holds. Let us choose a sequence of points $u_{n}$ from $[c, 0)$ such that $H_{1}\left(t_{0}, u_{n}\right)-H_{1}\left(t_{0}, c\right)=n(n=1,2$, $3, \cdots)$. Denote by $M_{i}\left(t_{0}, c, u_{n}\right)(i=1,2)$ the number of jumps of size $\in\left[c, u_{n}\right]$ of the process $X_{i}(t)$ before $t_{0}$. Then $M_{1}\left(t_{0}, c, u_{n}\right)$ is a Poisson variable with parameter equal to $n$, while $M_{2}\left(t_{0}, c, u_{n}\right)$ is a Poisson variable with parameter equal to $\mathrm{H}_{2}\left(t_{0}, u_{n}\right)-\mathrm{H}_{2}\left(t_{0}, c\right)$.

By the Chebyshev Inequality, we have for any $\delta>0$

$$
P_{X_{1}}\left(\left|\frac{M_{1}\left(t_{0}, c, u_{n}\right)}{n}-1\right| \geqq \delta\right) \leqq \frac{1}{\delta^{2} n^{2}} .
$$

By the Borel-Cantelli Lemma, since $\sum_{n=1}^{\infty} 1 / n^{2}<\infty$, relation (10) implies

$$
P_{X_{1}}\left(\lim _{n \rightarrow \infty} \frac{M_{1}\left(t_{0}, c, u_{n}\right)}{n}=1\right)=1 \text {. }
$$

Write $H_{2}\left(t_{0}, u_{n}\right)-H_{2}\left(t_{0}, c\right)=G\left(t_{0}, c, u_{n}\right)$. We have, as before, for any $\delta>0$

$$
P_{X_{2}}\left(\left|\frac{M_{2}\left(t_{0}, c, u_{n}\right)}{G\left(t_{0}, c, u_{n}\right)}-1\right| \geqq \delta\right) \leqq \frac{1}{\delta^{2} G^{2}\left(t_{0}, c, u_{n}\right)}
$$

By relations (8) and (9), we have

$$
\left(\rho_{-}-\varepsilon\right) n \leqq G\left(t_{0}, c, u_{n}\right) \leqq\left(\rho_{-}+\varepsilon\right) n
$$

hence

$$
\sum_{n=1}^{\infty} \frac{1}{G^{2}\left(t_{0}, c, u_{n}\right)}<\infty
$$

Again, by the Borel-Cantelli Lemma

$$
P_{X_{2}}\left(\lim _{n \rightarrow \infty} \frac{M_{2}\left(t_{0}, c, u_{n}\right)}{G\left(t_{0}, c, u_{n}\right)}=1\right)=1
$$

Now, if $\rho_{-}<1, \rho_{-}+\varepsilon<1$. Since, by (13),

$$
\frac{M_{2}\left(t_{0}, c, u_{n}\right)}{n} \leqq\left(\rho_{-}+\varepsilon\right) \frac{M_{2}\left(t_{0}, c, u_{n}\right)}{G\left(t_{0}, c, u_{n}\right)},
$$

we have by (15)

$$
P_{X_{2}}\left(\limsup _{n \rightarrow \infty} \frac{M_{2}\left(t_{0}, c, u_{n}\right)}{n}<1\right)=1
$$

Similarly, if $\rho_{-}>1, \rho_{-}-\varepsilon>1$. Since, again by (13),

$$
\frac{M_{2}\left(t_{0}, c, u_{n}\right)}{n} \geqq\left(\rho_{-}-\varepsilon\right) \frac{M_{2}\left(t_{0}, c, u_{n}\right)}{G\left(t_{0}, c, u_{n}\right)},
$$


we have by (15)

$$
P_{X_{2}}\left(\liminf _{n \rightarrow \infty} \frac{M_{2}\left(t_{0}, c_{0}, u_{n}\right)}{n}>1\right)=1 .
$$

It follows from (11), (17) and (19) that $P_{X_{1}} \perp P_{X_{2}}$.

If $\rho_{-}=\infty$, one gets the same result by interchanging the role of $H_{2}$ and $H_{1}$. The proof is analogous if $H_{1}\left(t_{0}, u\right)$ and $H_{2}\left(t_{0}, u\right)$ are not identically 0 for $u>0$. This remark completes the proof of Theorem 1 .

Proof of Theorem 2. Let $X_{1}(t)$ and $X_{2}(t)$ be separable, centered stable processes, without fixed discontinuity points, and let, for some $t_{0} \in(0,1], H_{1}\left(t_{0}, u\right)$ $\not \equiv H_{2}\left(t_{0}, u\right)$. If the exponent, say, $\alpha_{1}$ of the process $X_{1}(t)$ equals $2, H_{1}\left(t_{0}, u\right) \equiv 0$, while $H_{2}\left(t_{0}, u\right) \neq 0$. We have thus $P_{X_{1}} \perp P_{X_{2}}$, since the sample functions of $X_{1}(t)$ are continuous, with probability 1 , while those of $X_{2}(t)$ are almost all $\left(P_{X_{2}}\right)$ discontinuous. Let now $0<\alpha_{i}<2(i=1,2)$. If $H_{1}\left(t_{0}, u\right)=0$ for all $u<0$ while $H_{2}\left(t_{0}, u\right)=0$ for all $u>0$, then evidently $P_{X_{1}} \perp P_{X_{2}}$. If both $H_{1}\left(t_{0}, u\right)$ and $H_{2}\left(t_{0}, u\right)$ are not identically equal to 0 for, say, all $u<0$, we have

$$
\frac{H_{2}^{\prime}\left(t_{0}, u\right)}{H_{1}^{\prime}\left(t_{0}, u\right)}=k|u|^{\alpha_{1}-\alpha_{2}},
$$

where $k$ is some constant. Since $H_{1}\left(t_{0}, u\right) \neq \equiv H_{2}\left(t_{0}, u\right)$, we have either $\alpha_{1} \neq \alpha_{2}$, or $k \neq 1$, or both. Consequently, $\rho_{-}$equals either 0 , or $\infty$, or $k \neq 1$. By Theorem 1 , it follows $P_{X_{1}} \perp P_{X_{2}}$.

REMARK. The following example shows that there exist $L$-processes $X_{1}(t)$ and $X_{2}(t)$ such that $P_{X_{2}}$ is absolutely continuous with regard to $P_{X_{1}}$. Relations (3) and (4) are, of course, then satisfied.

EXAMPLE. Consider stationary, centered $L$-processes with $\gamma_{1}=\gamma_{2}=\sigma_{1}=\sigma_{2}=0$ and with

$$
H_{1}(u)= \begin{cases}0 & (u<0) \\ 2 \log u & (0<u \leqq 1 / 2) \\ 2 \log u / 2 & (1 / 2 \leqq u \leqq 2) \\ 0 & (u \geqq 2)\end{cases}
$$

and

$$
H_{2}(u)= \begin{cases}0 & (u<0), \\ 2 \log u & (0<u \leqq 1), \\ 0 & (u \geqq 1)\end{cases}
$$

By using Skorohod's [11] results, it is easy to check that $P_{X_{2}}$ is absolutely continuous with regard to $P_{X_{1}}$.

ACKNOWLEDGEMENT. The author is most grateful to the referee for his stimulating comments. 


\section{REFERENCES}

1. G. Baxter, A strong limit theorem for Gaussian processes, Proc. Amer. Math. Soc. 7 (1956), 522-527.

2. R. H. Cameron and T. W. Martin, The behavior of measure and measurability under change of scale in Wiener space, Bull. Amer. Math. Soc. 53 (1947), 130-137.

3. J. L. Doob, Stochastic processes, Wiley, New York, 1953.

4. J. Feldman, Equivalence and perpendicularity of Gaussian processes, Pacific J. Math. 8 (1958), 699-708.

5. B. V. Gnedenko and A. N. Kolmogorov, Limit distributions for sums of independent random variables, Addison-Wesley, Cambridge, Mass., 1954.

6. S. Kakutani, On equivalence of infinite product measures, Ann. of Math. 49 (1948), 214-224.

7. A. N. Kolmogorov, Grundbegriffe der Wahrscheinlichkeitsrechnung, Springer, Berlin, 1933.

8. N. Kryloff and N. Bogoliuboff, La théorie générale de la mesure dans son application à l'étude des systèmes dynamiques de la mécanique non-linéaire, Ann. of Math. 38 (1937), 65-113.

9. P. Lévy, Théorie de l'addition des variables aléatoires, Gauthier-Villars, Paris, 1937.

10. Yu. V. Prohorov, Convergence of random processes and limit theorems in probability theory, Teor. Verojatnost. i Primenen. 1 (1956), 177-238. (Russian)

11. A. V. Skorohod, On the differentiability of measures corresponding to stochatic processes with independent increments, Teor. Verojatnost. i Primenen. 2 (1957), 417-443. (Russian).

COLUMBia UNIVERSTTY,

New YoRK, New YoRK 